\title{
Learning from every death on an acute ward
}

\author{
Authors: Sundas Hasan, Billy Cheung, Mohammed Soliman and Carlo Prina
}

\section{Aims}

To launch a process ensuring review of all deaths on an acute medical unit using a standardised format and to generate learning points to share within the multidisciplinary team.

\section{Methods}

The process of formal mortality review on the acute medical ward at Homerton University Hospital started in 2015.

Every death was reviewed using a structured assessment form, completed by a junior doctor who was involved in the care, followed by the responsible consultant. This was then evaluated by an independent consultant and finally peer-reviewed in our quarterly mortality meeting. We used the confidential enquiry into sudden death of infants score to numerically document whether there was harm in each case. Learning points were generated where areas of potential improvement were identified. These were regularly presented to the multidisciplinary team to discuss and create action plans to improve future care. Following the first mortality meeting, we also measured the completion rates of treatment escalation plan (TEP) during inpatient admissions for 12 months and repeated this for the equivalent period in the following year.

\section{Results}

There were a total of 122 deaths between April 2015 and September 2017, of which all were formally reviewed, with results shown in Table 1.

The documented TEP for all acute medical admissions from October 2015 to September 2016 improved from 7.5\% to $18.3 \%$ within the same period in the subsequent year.

Table 1. Summary of results

\begin{tabular}{ll} 
CESDI score & Number of cases (\%) \\
0 & $85(69.7)$ \\
1 & $34(27.8)$ \\
2 & $3(2.5)$ \\
\hline CESDI = confidential enquiry into sudden death of infants.
\end{tabular}

Authors: Acute Care Unit, Homerton University Hospital, London, UK

\section{Conclusion}

Common learning themes emerged, all of which highlight the importance of early interventions. These include timely discussion regarding TEP, early recognition of the deteriorating patient and prompt initiation of palliative care plan where applicable.

Since our first mortality meeting, there have been significant improvements in the completion rates of documented TEP in the following year over the equivalent period.

We recognised the challenges in establishing a formal mortality review process and achieved this by creating an open and honest culture and objective review of all deaths. This process has also created an environment where we can confidently review each other's delivery of care. We invite regular feedback from everybody to improve this process. An overwhelming majority agreed that this has led to improved awareness in end-of-life care and has led to a better blame-free culture.

\section{Conflict of interest statement}

I, Dr Sundas Hasan, on behalf of my co-authors (Dr Billy Cheung, Dr Mohammed Soliman and Dr Carlo Prina) state that there is no conflict of interest to declare. 